Annuaire suisse de politique de développement

27-2 | 2008

Migration et développement: un mariage arrangé

\title{
Les migrations, une responsabilité partagée
}

\author{
Patricia A. Santo Tomas
}

\section{OpenEdition}

\section{Journals}

Édition électronique

URL : http://journals.openedition.org/aspd/503

DOI : 10.4000/aspd.503

ISSN : 1663-9669

\section{Éditeur}

Institut de hautes études internationales et du développement

\section{Édition imprimée}

Date de publication : 1 décembre 2008

Pagination : 93-99

ISBN : 978-2-940415-07-6

ISSN : 1660-5934

\section{Référence électronique}

Patricia A. Santo Tomas, "Les migrations, une responsabilité partagée », Annuaire suisse de politique de développement [En ligne], 27-2 | 2008, mis en ligne le 18 mars 2010, consulté le 07 septembre 2020. URL : http://journals.openedition.org/aspd/503 ; DOI : https://doi.org/10.4000/aspd.503 


\title{
Les migrations, une responsabilité partagée
}

\author{
Patricia A. Santo Tomas*
}

migrations ne datent pas d'hier aux Philippines. Les habitants de ce archipel - qui compte pas moins de 7107 îles disséminées à l'ouest du Pacifique - sont de tout temps partis s'installer ailleurs dans le pays quand la vie était trop dure ou qu'ils manquaient de perspectives sur place. Mais le Philippin ne se laisse pas enfermer dans les frontières nationales: selon des témoins de l'époque, les navires portugais ou espagnols qui exploraient la région au cours du $\mathrm{XV}^{\mathrm{e}}$ siècle ont ramené chez eux des Philippins fascinés par des histoires de pays lointains. Et c'est toujours pareil aujourd'hui. Durant le premier semestre de 2007, plus d'un demi-million de Philippins sont partis outre-mer trouver des emplois qui les séparent de leur famille et des amis pour de longues périodes. Ce sont les travailleurs émigrés, ou overseas Filipino workers (OFW). Ce chiffre n'inclut pas la multitude de Philippins - touristes, étudiants, hommes d'affaires et autres voyageurs - qui contribuent à donner un caractère très cosmopolite à la culture de ce pays.

Ce mouvement migratoire est stimulé par la demande mondiale de ressources humaines, un phénomène alimenté dès le milieu des années 1970 par les recettes pétrolières qui ont fait du Moyen-Orient un pôle d'attraction pour les travailleurs du monde entier. Il y a aussi la sénescence qui affecte progressivement les pays développés, sans doute en réaction aux prédictions catastrophistes sur la probable explosion démographique menaçant d'étouffer notre monde aux dimensions figées. Par ailleurs, l'univers virtuel qu'offre Internet ainsi que les progrès des transports et des communications donnent au reste du monde une idée de ce que fait l'autre moitié de la planète.

Tous ces facteurs exercent bien entendu une influence sur la propension des Philippins à émigrer. Par ailleurs, un taux de scolarisation qui dépasse 90\%, l'importance accordée aux études supérieures ${ }^{1}$ et une économie en expansion - avec au moins un téléphone mobile dans le moindre taudis - encouragent les gens à tenter leur chance ailleurs.

Les disparités démographiques, l'existence de poches de troubles et les répercussions politiques du terrorisme expliquent en partie pourquoi les migrations sont

* Présidente de la Development Bank of the Philippines (DBP) et ancienne secrétaire du Department of Labor and Employment (DOLE). De mai 1982 à septembre 1987, Patricia A. Santo Tomas a été directrice de la Philippine Overseas Employment Administration (POEA).

Le présent article est basé sur un exposé fait le 30 octobre 2007 à Berne, dans le cadre de la conférence annuelle de la Division politique IV du Département fédéral des affaires étrangères (DFAE). Il met en lumière l'évolution récente selon le point de vue d'un pays du Sud qui est lui-même une source de mouvements migratoires, et fournit un exemple de concrétisation d'une politique active en matière de migrations.

1 Le pays comptait en 2000 plus de 2,6 millions d'étudiants enregistrés (P. Santo Tomas, «Filipinos Working Overseas: Opportunity and Challenge », in International Organization for Migration [IOM], World Migration 2005: Costs and Benefits of International Migration, Geneva, IOM, 2005, pp. 239251). 
devenues un sujet brûlant. Parallèlement, l'évolution des transports, des communications et des réseaux sociaux transnationaux facilite la mobilité à l'échelle planétaire. Il faut que les instances bien au courant du phénomène lui accordent sereinement l'importance qu'il mérite, avant que la problématique migratoire ne devienne un nouveau foyer de crise dans les relations internationales.

\section{Migration temporaire, le mode dominant}

Les migrants philippins peuvent être classés selon différents critères - par exemple la durée du séjour à l'étranger, qui peut être temporaire ou permanent (ou définitif). On qualifie de migrants permanents les immigrants ou les résidents permanents dans un pays étranger dont le séjour ne dépend pas d'un contrat de travail, tandis que les migrants temporaires sont des personnes qui ont un emploi à l'étranger et sont censées retourner dans leur pays d'origine à l'échéance du contrat de travail ${ }^{2}$.

Le statut, régulier ou irrégulier, constitue aussi un critère. Les migrants réguliers sont ceux dont les papiers sont en règle et attestent leur statut de migrant temporaire ou permanent. Les migrants sans papiers sont soit sans papiers en règle, soit sans permis de séjour ou de travail valide, ou encore ils séjournent dans un pays étranger au-delà de la période autorisée.

Des statistiques nationales indiquent également si le migrant va aux Etats-Unis ou en Asie (destination), s'il est homme ou femme (sexe), cuisinier ou médecin (contrat spécifique), de profession libérale ou employé (statut professionnel), sur terre ou sur mer (zone d'emploi).

Selon des estimations officielles, l'effectif total des Philippins à l'étranger en 2006 se chiffrait à 8233172 personnes $^{3}$, dont $43 \%$ séjournaient en Amérique du Nord (voir tableau 1). Près de la moitié des OFW (46\%) sont des migrants temporaires, et $11 \%$ sont des irréguliers et probablement temporaires (voir tableau 2). La catégorie des migrants réguliers comprend 274497 marins, lesquels constituent environ $7 \%$ des travailleurs temporaires.

\section{Tableau 1: Philippins à l'étranger par destination (effectifs de migrants temporaires et permanents), 2006}

\begin{tabular}{lc}
\hline Région de destination & Part du total mondial (8,2 millions) \\
\hline Amérique du Nord et du Sud & $43 \%$ (3,6 millions) \\
\hline Asie occidentale (y compris Moyen-Orient) & $22 \%(1,8$ million) \\
\hline Asie de l'Est et du Sud & $15 \%$ \\
\hline Europe & $11 \%$ \\
\hline Océanie (Australie, Nouvelle-Zélande, etc.) & $4 \%$ \\
\hline Afrique & $1 \%$ \\
\hline Travailleurs maritimes & $3,5 \%$ \\
\hline
\end{tabular}

Source: Commission on Filipinos Overseas, "Stock Estimate of Overseas Filipinos as of December 2006", <http://www.cfo. gov.ph>>Statistics.

2 Cette définition ne correspond pas à l'acception internationale courante, selon laquelle les migrations temporaires durent plus de trois mois et moins d'une année.

3 L'Office national de la statistique de la République des Philippines estime que le pays comptait 87 millions d'habitants en 2006 (<http://www.census.gov.ph>), ce qui signifie que $10 \%$ environ de sa population sont des migrants temporaires ou permanents. Ces proportions sont similaires en Suisse, mais des volumes démographiques bien différents rendent les deux pays difficilement comparables. 
Tableau 2: Philippins à l'étranger par type de migration, 2006

\begin{tabular}{lc}
\hline Type de migration & Part du total mondial \\
\hline Migration temporaire & $46 \%$ (3,8 millions) \\
\hline Migration permanente & $43 \%$ (3,5 millions) \\
\hline Migration irrégulière & $11 \%(0,9$ million $)$ \\
\hline
\end{tabular}

Source: Commission on Filipinos Overseas, "Stock Estimate of Overseas Filipinos as of December 2006", <http://www.cfo. gov.ph>>Statistics.

La Philippine Overseas Employment Administration (POEA) enregistre annuellement une moyenne de 900000 contrats de travailleurs partant pour l'étranger. Il est intéressant de relever que les Etats-Unis voient affluer le plus grand nombre d'immigrants permanents, et seulement $1,5 \%$ de migrants philippins temporaires. En 2006, l'Arabie saoudite ne comptait que 247 immigrants permanents sur un total de 3,6 millions de Philippins établis de façon permanente à l'étranger.

Au cours des vingt dernières années, l'émigration temporaire des Philippins a progressé beaucoup plus rapidement que leur émigration permanente. Les emplois à l'étranger ont reculé en 1989, en 1990, en 1995 et en 2003. L'émigration permanente présente un tableau plus varié, avec des baisses dans les années 1989, 1991, 1994, 1995, 1997, 1998 et 2003. Il convient aussi de souligner que l'émigration permanente n'a jamais atteint la barre des 100000 personne par année, alors que les départs temporaires ont régulièrement dépassé cette limite ces vingt dernières années - et même passé le cap du million en 2006.

Il apparaît ainsi que dans le cas des Philippines, le mode d'émigration dominant présente un caractère temporaire (migration sous contrat de travail).

\section{Tendances récentes aux Philippines}

Le marché international de l'emploi a passablement évolué depuis le milieu des années 1970 et le boom de la construction lié au développement de certains pays du Moyen-Orient. On constate que les caractéristiques de la migration temporaire sont en train de changer: alors que le total des emplois à l'étranger reste à la hausse, les contrats renouvelés prennent le pas $(60 \%)$ sur les nouveaux contrats de travail qui se chiffrent à $40 \%$ en moyenne. Le profil statistique des migrants philippins s'en trouve également modifié dans le sens où les jeunes d'autrefois sont aujourd'hui des travailleurs de 30 ans et plus.

Alors qu'au début les Philippins partis travailler à l'étranger étaient pour la plupart occupés dans la production, cette catégorie est aujourd'hui dépassée par les travailleurs du tertiaire, suivis de près par les professions libérales, techniques et médicales.

Autrefois, les Philippins travaillaient comme entrepreneurs généraux et passaient des contrats clés en main, tandis qu'actuellement les acteurs centraux sont des intermédiaires - surtout des recruteurs étrangers ou locaux - fournisseurs de main-d'œuvre.

La répartition des sexes change aussi: les ouvriers, main-d'œuvre très demandée dans les années 1970, sont aujourd'hui dépassés en nombre par les femmes. Celles-ci représentaient $20 \%$ de l'émigration à cette époque, et $60 \%$ à $90 \%$ 
actuellement selon la catégorie d'emplois considérée. Les femmes ont clairement l'avantage dans les métiers de la santé, l'enseignement, l'hôtellerie et la restauration.

La première destination est toujours le Moyen-Orient, mais l'Asie se rapproche en deuxième position. Par pays, l'Arabie saoudite reste en tête à la fois pour les premiers contrats et les renouvellements (voir tableau 3). Côté premiers emplois on observe cependant l'apparition de nouveaux noms - Corée, Etats-Unis, Liban et Japon - dans les dix premières destinations.

Tableau 3: Répartition des OFW selon les dix premières destinations (premiers contrats de travail et renouvellements), 2005-2006

\begin{tabular}{lrrrr}
\hline Destinations & 2005 & 2006 & $\% 2005$ & $\% 2006$ \\
\hline Arabie saoudite & 194350 & 223459 & 26,2 & 28,4 \\
\hline Emirats arabes unis & 82039 & 99212 & 11,1 & 12,6 \\
\hline Hongkong & 98693 & 96929 & 12,3 & 12,3 \\
\hline Koweït & 40306 & 47917 & 5,4 & 6,1 \\
\hline Oatar & 31421 & 45795 & 4,2 & 5,8 \\
\hline Taiwan & 46737 & 39025 & 6,3 & 5,0 \\
\hline Singapour & 28152 & 28369 & 3,8 & 3,6 \\
\hline Italie & 21267 & 25413 & 2,9 & 3,2 \\
\hline Royaume-Uni & 16930 & 16926 & 2,3 & 2,1 \\
\hline Corée & 9975 & 13984 & 1,3 & 1,8 \\
\hline Autres destinations & 179762 & 151041 & 23,1 & 19,2 \\
\hline Total destinations terrestres & 740632 & 788070 & 100 & 100 \\
\hline
\end{tabular}

Source: Philippine Overseas Employment Administration (POEA), OFW Global Presence: A Compendium of Overseas Employment Statistics: 2006, <http://www.poea.gov.ph>.

\section{Questions cruciales et implications sociales}

Les problèmes liés au travail à l'étranger n'ont guère changé au cours du temps. Il y a celui de la fuite des cerveaux, une fois de plus concentré sur le personnel soignant. C'est dès les années 1950 que des professionnels philippins de la santé ont émigré vers des pays plus développés, suscitant le reproche que les pays en développement subventionnaient ainsi des systèmes de santé étrangers. Les mouvements migratoires des Philippins ont été cycliques dans ce secteur, avec des fluctuations vers le haut ou vers le bas par périodes de sept ans. On voit ainsi que la demande internationale a considérablement influencé les choix de carrière aux Philippines bien avant l'avènement d'Internet, avec des excédents ou des pénuries d'effectifs dus au décalage des études de licence. Cette fois-ci, les formations les plus courues ont été celles d'infirmière et d'informaticien - la première, manifestement, avec un œil sur le marché du travail étranger, tandis que la seconde est liée au phénomène de sous-traitance des opérations commerciales pour les processus d'arrière-plan, les centres d'appel, l'animation et d'autres services informatiques réalisés localement.

Le deuxième sujet de préoccupation, déjà évoqué plus haut, est la féminisation de la main-d'œuvre philippine à l'étranger. Cela s'explique d'un côté par la nature de la demande, et aussi par le fait que les femmes sont majoritaires dans les formations de base aussi bien qu'au niveau des études supérieures. Cette évolution a des répercussions importantes sur les rôles sociaux des deux sexes - 
notamment celui de la Philippine devenue soutien de famille - et sur les relations entre générations.

La problématique sociale a aussi son importance: on trouve des Philippins dans 197 pays et territoires de tous les continents, et il peut constamment y avoir des incompatibilités entre les immigrés et la culture ou les coutumes locales, sans parler de la religion ou du régime politique. Les contacts entre cultures et conceptions juridiques différentes sont une source de malentendus. Même avec un séminaire d'information et de sensibilisation culturelle avant le départ ${ }^{4}$, tout le monde ne parvient pas à assimiler certaines particularités culturelles. En plus de son corps diplomatique, l'Etat philippin entretient un réseau mondial de 200 attachés du travail et assistants sociaux qui s'occupent du bien-être de ses citoyens expatriés.

La réintégration des migrants pose bien des problèmes aux Philippines. Mis à part, peut-être, ceux qui s'établissent aux Etats-Unis, au Canada, en Australie ou au Japon, la grande majorité des Philippins rentrent chez eux à la fin de leur contrat de travail, même si celui-ci a été renouvelé plusieurs fois. Ceux qui s'en tirent bien ont pu investir dans la formation de leurs enfants et dans les habitations de leurs familles restées au pays. Mais beaucoup reviennent sans économies ou bien gaspillent leur argent pour des produits de luxe. Certains n'ont même ni plan de retraite ni assurance médicale. Les efforts de réintégration entrepris par le gouvernement philippin se concentrent sur le retour anticipé, la formation en entreprise, la prévoyance vieillesse et des investissements dans le capital humain.

Se pose aussi la question de l'alternative entre dépendre de l'argent versé depuis l'étranger par les migrants du pays ou stimuler davantage l'économie locale. Alors que l'afflux financier depuis l'extérieur s'est considérablement accru au cours des ans, le gouvernement philippin a bien suivi en termes de budgets consacrés à l'infrastructure et au capital humain. Les versements de travailleurs philippins ont augmenté de $26 \%$ entre 2004 et 2005 pour atteindre 10,7 milliards de dollars, dont 6,4 milliards envoyés par des migrants aux Etats-Unis. Mais cette idée de dépendance reste un sujet de controverses politiques et scientifiques: quels sont, notamment, les effets des versements en provenance de l'étranger sur l'économie locale? Alors que ces apports contribuent à soulager la pauvreté à l'échelle des familles, leur effet macroéconomique de croissance durable est mis en doute par certains auteurs qui stigmatisent des inégalités grandissantes et la situation de dépendance des pays bénéficiaires des transferts de fonds.

On exagère parfois le rôle des Philippines en tant qu'exportateur de maind'œuvre, alors même que les travailleurs de ce pays n'auraient pas l'occasion d'émigrer en l'absence de demande. Les pays importateurs le sont en fonction de leurs besoins. Le gouvernement philippin a ainsi mis en place une réglementation très claire, un mécanisme de protection détaillé et un programme de réintégration qui ne dépend pas des pays qui recourent à notre capital humain. Les Philippines considèrent la migration comme une responsabilité partagée, une politique qu'elles suivent au mieux de leurs ressources limitées.

4 Les candidats à des emplois à l'étranger doivent suivre un pre-departure orientation seminar (PDOS) qui les instruit sur les lois et les coutumes du pays de destination (cf. P. Santo Tomas, op. cit.). 
Il est important de savoir que la possibilité d'émigrer temporairement s'offre aux travailleurs philippins depuis les années 1970. Et alors que nous avons fixé des normes pour le travail local, nous en avons également pour les emplois à l'étranger. Lorsque ceux-ci ont été officialisés pour combattre le chômage, le gouvernement a pris de nouvelles dispositions légales, puis créé deux instances répondant aux besoins des travailleurs à l'étranger: la Philippine Overseas Employment Administration (POEA) et la Overseas Workers Welfare Administration (OWWA). Le Code du travail a servi de cadre juridique au moment où le programme d'emplois à l'étranger démarrait en 1976. Un certain nombre de lois ont été promulguées depuis lors pour régler les détails de la migration temporaire.

Le gouvernement philippin réglemente, mais offre aussi des services de médiation lorsqu'un autre gouvernement le demande ${ }^{5}$. Cela s'est produit au début de la migration à destination de l'Arabie saoudite, même si ces accords se limitent désormais aux hôpitaux d'Etat de ce pays. Le Royaume-Uni a passé un arrangement similaire concernant le recrutement d'infirmières pour sa Sécurité sociale (National Health Service, NHS). Des accords semblables ont été signés avec la Corée et le Japon. Lorsque c'est possible, le gouvernement philippin préfère laisser les activités de recrutement au secteur privé.

Considérant qu'une protection efficace des travailleurs à l'étranger est dans l'intérêt de tous les acteurs concernés - la personne migrante, son pays d'origine et le pays d'accueil -, le gouvernement philippin a mis sur pied un programme de suivi qui englobe toutes les phases du processus, depuis la candidature à un poste et la formation adéquate jusqu'à la réintégration lors du retour. Il pratique un système de licences attribuées à plus d'un millier d'agences de recrutement. Les employeurs sont agréés par le gouvernement, et sanctionnés s'ils violent les conditions des contrats de travail. Un recruteur qui déroge aux règles perd sa licence. Quant aux travailleurs eux-mêmes, ils sont tenus, avant leur départ, de subir un examen médical et psychologique ainsi qu'un contrôle de leurs qualifications. Leurs contrats sont enregistrés par la Philippine Overseas Employment Administration dans une banque de données, ce qui permet de retrouver au besoin leurs coordonnées.

Les travailleurs sont couverts par une assurance qui s'étend à la durée du contrat plus deux mois après leur retour. En cas de situation catastrophique sur leur lieu de travail (une guerre comme en Irak, une épidémie telle que le SRAS), les Philippins peuvent être rapatriés par leur gouvernement ou évacués dans un pays tiers. On tient aussi un registre des employeurs, et ceux qui ont fait subir des mauvais traitements à leurs salariés philippins n'ont plus l'autorisation d'en engager. Le passeport philippin donne aussi la liste des pays dans lesquels il est interdit d'employer des Philippins.

Dans les principaux territoires où travaillent des Philippins, les OFW trouvent des centres de consultation et de formation continue, celle-ci étant dispensée

5 On se réfère ici aux activités de recrutement et de formation des travailleurs philippins pratiquées par des instances gouvernementales des pays d'origine et d'accueil (et non à des agences privées de recrutement et de placement). 
durant leurs week-ends de congé. Les Philippins bénéficient d'une représentation juridique partout où ils se trouvent, même dans les situations où ils semblent avoir contrevenu aux lois du pays qui les accueille. Ceux qui tombent malades et requièrent des soins médicaux chez eux peuvent être rapatriés pour être hospitalisés aux Philippines.

Le gouvernement s'emploie également à passer des accords bilatéraux pour améliorer les mécanismes de protection des travailleurs philippins. Dans quatre Etats au moins, ces accords comprennent des conditions de travail (salaires inclus) comparables à celles dont bénéficient les ressortissants du pays considéré.

Dans cette optique de responsabilité partagée, les Philippines s'efforcent de protéger leurs travailleurs mais aussi de veiller à ce que ceux-ci respectent les coutumes et les lois de leur pays d'accueil temporaire. Notre gouvernement demande en échange que l'on protège ses OFW contre quiconque porte atteinte à leurs droits.

\section{Conclusion}

L'emploi dépend en fin de compte des lois de l'offre et de la demande, que ce soit à l'échelle nationale ou internationale, mais il exige aussi une réglementation transparente pour la protection personnelle et sociale des travailleurs, quel que soit leur statut de résidence. Estimant que la mobilité transnationale du travail peut être bénéfique aussi bien pour les pays d'accueil que pour les travailleurs et leur pays d'origine, la gouvernement philippin a mis en place un système de règles sur l'emploi à l'étranger couvrant toutes les phases du processus, depuis la formation précédant le départ jusqu'à la réintégration après le retour. La raison d'être de ces mesures, c'est qu'un manque de protection comporte de sérieux dangers non seulement pour les migrants, mais aussi pour la société du pays d'accueil dans son ensemble. Il est avéré que l'absence de règles précises concernant les droits des migrants ne fait qu'amplifier de graves problèmes tel que le travail clandestin, synonyme de concurrence déloyale, dumping social et fraude fiscale, trafic humain, activités criminelles et insécurité.

De plus en plus, on se rend compte qu'il ne suffit pas de réglementer unilatéralement les flux migratoires, qu'il est nécessaire d'encourager la coopération internationale et de trouver des solutions constructives incluant pays d'origine et pays d'accueil. Dans cet esprit, une politique de réglementation systématique et bien intégrée peut favoriser le dialogue et le partage des responsabilités entre Etats directement concernés par la migration, ainsi qu'à l'échelle multilatérale. La communauté internationale pourra s'inspirer de ces mécanismes aussi bien pour les mouvements temporaires que pour l'émigration définitive. 